\title{
The New Photometric Observations for Transiting Exoplanet HAT-P-24b
}

\author{
Xiao-Bin Wang ${ }^{1,2}$, Andrew Collier Cameron ${ }^{3}$ and Sheng-Hong Gu ${ }^{1,2}$ \\ ${ }^{1}$ National Astronomical Observatories/Yunnan Observatory \\ Chinese Academy of Sciences, Kunming 650011, China \\ email: wangxb@ynao.ac.cn \\ ${ }^{2}$ Key Laboratory for the Structure and Evolution of Celestial Objects \\ Chinese Academy of Sciences, Kunming 650011, China \\ ${ }^{3}$ School of Physics and Astronomy, University of St Andrews \\ North Haugh, St Andrews, Fife KY16 9SS, UK
}

\begin{abstract}
The transiting exoplanetary system HAT-P-24 was observed by using CCD cameras at Yunnan Observatory and Hokoon Astronomical Centre, China in 2010 and 2012. Three new transit light curves are analyzed by means of MCMC technique, and the new physical parameters of the system are derived, which are compatible with the old ones in the discovery paper. The orbital period of HAT-P-24b is refined and no obvious TTV signal can be found from five transit events during 2010-2012.
\end{abstract}

Keywords. exoplanetary system, transit, individual:HAT-P-24

\section{Introduction}

Photometric observation of transiting exoplanetary system can provide us the size and mass of the planet, which are important for understanding the formation and evolution of exoplanet (Charbonneau et al. 2000). The monitoring for known transiting exoplanets can let us know whether there are other planets in the same systems, through analyzing transit timing variation (TTV) and transit duration variation (TDV) (Agol et al. 2005, Holman \& Murray 2005, Kipping 2009a, Kipping 2009b). Furthermore, more precise observations can permit us to improve the physical parameters of transiting systems, which is helpful to understand detailed aspects relative to exoplanet formation and evolution. Therefore, photometric follow-up observations for known exoplanetary systems are also important besides discovering new ones by photometric survey.

\section{Observations and data reduction}

HAT-P-24b was discovered by Kipping et al. (2010), and it is an inflated hot-jupiter with a mass of $0.681 M_{\mathrm{J}}$, radius of $1.243 R_{\mathrm{J}}$ and small eccentricity of $e=0.052$. On Dec. 28 , 2010 and Mar. 15, 2012, HAT-P-24 was observed by using an Andor CCD camera with a $2 \mathrm{~K} \times 2 \mathrm{~K}$ chip attached to $1 \mathrm{~m}$ telescope of Yunnan Observatory, China, and an Apogee $\mathrm{CCD}$ camera with a $3 \mathrm{~K} \times 3 \mathrm{~K}$ chip attached to $50 \mathrm{~cm}$ telescope of Hokoon Astronomical Centre, Hongkong, China. During the observations, R filter was used. All observed CCD images of both instruments are reduced in the standard way using the IRAF package. The instrumental magnitude values of the objects in the reduced images are measured using APPHOT sub-package of IRAF. Because normally the transit signal of an exoplanet is weak, we use coarse de-correlation and SYSREM methods to correct the systematic errors in the photometric data (Collier Cameron et al. 2006, Tamuz et al. 2005). The final light curves of the transit events for the system HAT-P-24 are shown in Fig. 1. 

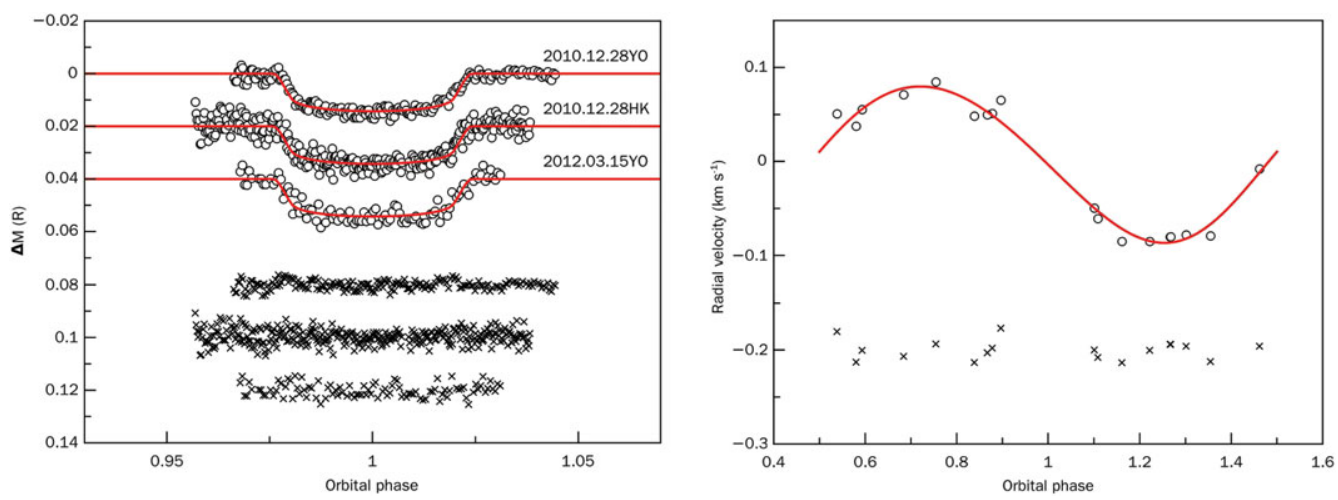

Figure 1. Observed light curves, radial velocity curve and relative model fitting. For the sake of better display, two light curves and the residuals between observations and theoretical curves are offset by constants.

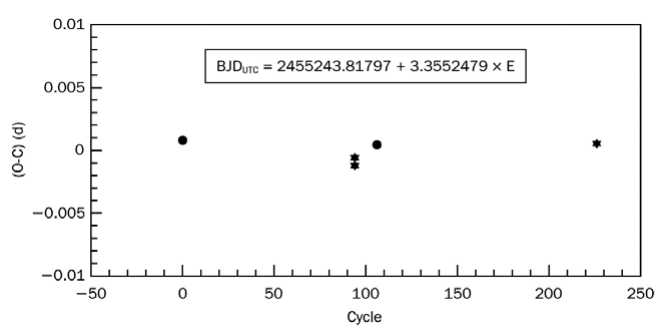

Figure 2. The (O-C) diagram for orbital period study of HAT-P-24 system.

\section{Light curve analysis}

In order to derive the system parameters of HAT-P-24, we use Markov Chain Monte Carlo (MCMC) technique (Collier Cameron et al. 2007) to analyze the three above transit light curves and published radial velocity curve (Kipping et al. 2010) simultaneously. The transit light curve is modeled using small planet approximation (Mandel \& Agol 2002), and the 4-coefficient limb-darkening law is used (Claret 2000). The best system parameters are derived from the median values of the posterior probability distribution.

We first analyzed three new transit light curves and Kipping et al. (2010)'s radial velocity curve simultaneously to get a global solution for photometric and radial velocity observations. This gives us an orbital period $P=3.35524825$ days. In the second step, we modeled the individual transit light curves and the radial velocity curves to determine the accurate epoch of mid-transit for each transit events. Based on 5 available epochs of mid-transits obtained from high precision observations, we have derived an improved linear ephemeris formula $B J D_{U T C}=2455243.81797+3.3552479 \times E$, and the relative (OC) diagram is shown in Fig. 2. Finally, using the new orbital period, the best solution for HAT-P-24 system is derived by the MCMC procedure through combining three new transit light curves with Kipping et al. (2010)'s radial velocity curve, which is listed in Table 1, the relative model fitting is shown in Fig. 1.

\section{Summary}

Based on above analysis for the new photometric observations and published radial velocity data, we have revised the physical parameters of transiting system HAT-P-24. The new result is compatible to the old one (Kipping et al. 2010). The orbit exhibits 
Table 1. System parameters and $1 \sigma$ error limits derived from the MCMC analysis.

\begin{tabular}{llll}
\hline Parameter & Symbol & Value & Unit \\
\hline Transit epoch & $T_{0}$ & $2455629.67053 \pm 0.00034$ & days \\
Orbital period & $P$ & $3.3552479 \pm 0.0000062$ & days \\
Planet/star area ratio & $\left(R_{p} / R_{*}\right)^{2}$ & $0.01129 \pm 0.00016$ & \\
Transit duration & $t_{T}$ & $0.1535 \pm 0.0022$ & days \\
Impact parameter & $b$ & $0.2367_{-0.0845}^{+0.0875}$ & $R_{*}$ \\
Stellar reflex velocity & $K_{1}$ & $0.0832^{+0.0033}$ & $\mathrm{~km} / \mathrm{s}$ \\
Orbital eccentricity & $e$ & $0.064_{-0.020}^{+0.023}$ & \\
Longitude of periastron & $\omega$ & $-125.39_{-37.56}^{+287.73}$ & degrees \\
Orbital inclination & $i$ & $88.217_{-0.693}^{+0.716}$ & degrees \\
Stellar mass & $M_{*}$ & $1.195 \pm 0.012$ & $M_{\odot}$ \\
Stellar radius & $R_{*}$ & $1.321 \pm 0.063$ & $R_{\odot}$ \\
Orbital semi-major axis & $a$ & $0.04655_{-0.00016}^{+0.00015}$ & $\mathrm{AU}$ \\
Planet radius & $R_{p}$ & $1.364 \pm 0.068$ & $R_{\mathrm{J}}$ \\
Planet mass & $M_{p}$ & $0.691 \pm 0.027$ & $M_{\mathrm{J}}$ \\
Planet density & $\rho_{p}$ & $0.272_{-0.034}^{+0.044}$ & $\rho_{\mathrm{J}}$ \\
Planet temperature & $T_{e q}$ & $1637_{-37}^{+35}$ & $\mathrm{~K}$ \\
\hline
\end{tabular}

a small eccentricity $e=0.064$, slightly larger than the old one $e=0.052$. The new orbital period is slightly longer than the one reported by Kipping et al. (2010) which should be more accurate than the old one because 5 epochs of mid-transits with complete transit light curves have been used in the analysis. The radius of planet $R_{p}=1.364 R_{\mathrm{J}}$ is larger than the old one. No obvious TTV signal can be found from the present (O-C) analysis of orbital period.

\section{Acknowledgements}

We thank the $1 \mathrm{~m}$ telescope operators and some graduate students of Yunnan Observatory for their support and help during our observations. This work was supported by the National Natural Science Foundation of China (No.10873031), Chinese Academy of Sciences (KJCX2-YW-T24) and the special grant from Sik Sik Yuen of Hong Kong, China.

\section{References}

Agol, E., Steffen, J., Sari, R., et al. 2005, MNRAS, 359, 567

Charbonneau, D., Brown, T. M., Latham, D. W., et al. 2000, ApJ, 529, L45

Claret, A. 2000, A\& A, 363, 1081

Collier Cameron, A., et al. 2006, MNRAS, 373, 799

Collier Cameron, A., Wilson, D. M., West, R. G., et al. 2007, MNRAS, 380, 1230

Holman, M. J. \& Murray, N. W. 2005, Science, 307, 1288

Kipping, D. M. 2009, MNRAS, 392, 181

Kipping, D. M. 2009, MNRAS, 396, 1797

Kipping, D. M., Bakos, G. A., Hartman, J., et al. 2010, ApJ, 725, 2017

Mandel, K. \& Agol, E. 2002, ApJ, 580, L171

Tamuz, O., et al. 2005, MNRAS, 356, 1466 